\title{
The Relationship between CT Severity Score and Capillary Blood Oxygen Saturation in Patients with COVID-19 Infection
}

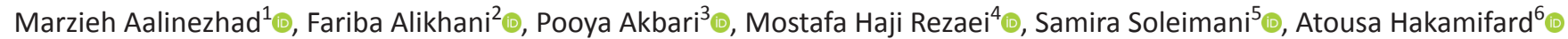

\begin{abstract}
Background: COVID-19 pneumonia is responsible for the latest pandemics. Usage of pulmonary computed tomography (CT) scan is known to be an important method in the diagnosis of COVID-19. Here, we aimed to evaluate the relationship between CT severity score and capillary blood oxygen saturation in patients with COVID-19 infection.

Patients and methods: This is a cross-sectional study performed in 2020 on patients with COVID-19 infection. All patients underwent pulmonary CT scan and CT severity score was calculated. The initial capillary oxygen saturation by the time of admission was also collected. Data were collected and analyzed.

Results: A total number of 270 patients diagnosed with COVID-19 infection entered the study. The initial mean capillary oxygen saturation was $89.65 \pm 8.09 \%$. The mean score in patients was $15.16 \pm 8.08$. We also indicated that 27 patients had hypoxia by the time of admission and these patients had significantly higher CT severity scores $(p=0.001)$. Diabetes mellitus $(p=0.001)$, hypertension $(p=0.001)$, and chronic obstructive pulmonary disease (COPD) $(p=0.03)$ and totally, having an underlying disease $(p<0.001)$ were significantly associated with decreased capillary oxygen saturation. Patients with hypertension $(p=0.001)$ and patients with the previous medical disease $(p=0.01)$ had significantly higher CT severity scores than others. There was a significant reverse relationship between CT severity score and capillary oxygen saturation $(r=-0.44$, $p<0.001$ ).

Conclusions: We showed that there was a significant reverse relationship between $\mathrm{CT}$ severity score and capillary oxygen saturation which has great clinical importance. Furthermore, we indicated that previous medical history could also play an important role in CT severity score. Keywords: Computed tomography, COVID-19, CT severity score, Hypoxia.

Indian Journal of Critical Care Medicine (2021): 10.5005/jp-journals-10071-23752
\end{abstract}

\section{INTRODUCTION}

Coronavirus is a large family of viruses that includes the common cold virus and the agent of a severe acute respiratory syndrome (SARS). Coronavirus disease (COVID-19) is an infectious disease caused by a newly discovered coronavirus. ${ }^{1,2}$ The novel coronavirus is a new respiratory coronavirus that started in late 2019 and early 2020 in Hubei province and Wuhan city of China and has caused a new crisis for the world. ${ }^{3}$ The disease is called "acute respiratory syndrome coronavirus 2" (SARS-CoV-2), and the World Health Organization (WHO) named the virus COVID-19 after the death toll exceeded $1,000 .{ }^{4}$ The virus is transmitted from animals to humans and, due to the behavior of the virus and its highly contagious nature, was quickly spread through human-to-human contact all around the globe. ${ }^{5,6}$

The SARS-CoV-2 virus started in Wuhan, Hubei Province, China, in early December 2019, and then gradually spread to other parts of the world, causing a pandemic. ${ }^{7}$ The most common clinical manifestations of COVID-19 pneumonia are fever, fatigue, dry cough, myalgia, shortness of breath, and gastrointestinal complications, such as diarrhea and vomiting. . $^{8,9}$ Based on the evidence, the manifestations of the disease vary in individuals, but the main manifestations include cough, upper airway inflammation, myalgia, headache, acute respiratory distress syndrome (ARDS), decreased blood $\mathrm{O}_{2}$ saturation, and pulmonary involvement in imaging studies. ${ }^{10}$ It has also been declared that these pulmonary involvements are the most important prognostic factors in patients with COVID-19infection.
1-3,5 Deparment of Radiology, Isfahan University of Medical Sciences, Isfahan, Isfahan, Iran

${ }^{4}$ Infectious Diseases Research Center, Kashan University of Medical Sciences, Kashan, Iran.

${ }^{6}$ Department of Infectious Diseases, School of Medicine, Isfahan University of Medical Sciences, Isfahan, Isfahan, Iran

Corresponding Author: Fariba Alikhani, Department of Radiology, Isfahan University of Medical Sciences, Isfahan, Isfahan, Iran, Phone: +989132027583, e-mail: Fariba.Alikhani@med.mui.ac.ir

How to cite this article: Aalinezhad M, Alikhani F, Akbari P, Haji Rezaei M, Soleimani S, Hakamifard A. The Relationship between CT Severity Score and Capillary Blood Oxygen Saturation in Patients with COVID-19 Infection. Indian J Crit Care Med 2021;25(3):279-283.

Source of support: This study was supported by a research grant from the Vice Chancellor for Research of Isfahan University of Medical Sciences Conflict of interest: None

Lung computed tomography (CT) scan is an important method in the diagnosis of pulmonary abnormalities, which also has a very valuable role in screening the patients suspected of infections as well as diagnosis and clinical classifications of these patients. ${ }^{11}$ Using CT scans, physicians are also able to follow-up patients after discharge from the hospital. In previous studies, imaging findings of lung infection caused by COVID-19 have been described in CT 
scans. The most common patterns of this infection are bilateral and multilobar ground-glass opacities. ${ }^{12,13}$ It has also been shown that the peripheral and lower lobes of the lung are mostly affected. Further studies also showed other radiological patterns, such as crazy paving pattern, airway change, and reverse halo sign for patients infected with COVID-19.14,15

Some studies have also emphasized the relationship between CT scan findings and the clinical condition of patients explaining the possible role of lung CT scan in determining the severity and spread of the disease. CT severity score is a semi-quantitative scoring system that is developed to determine the severity and extent of pulmonary involvement in viral pneumonia. ${ }^{16}$ Some previous studies have suggested that this scoring system could be used to quantify the spread of COVID-19 pneumonia in the lungs of patients and also could be used to evaluate the clinical conditions of patients. ${ }^{17}$ By the time of admission of patients with COVID19 infection to the hospital, the measurement of blood oxygen levels with a pulse oximetry device is performed for almost all the patients. ${ }^{18}$ Hypoxia could be indicated in patients using this device, which indicates a higher severity of the disease and a greater need for intensive care. ${ }^{19}$ Very few studies have evaluated the possible relationships between quantitative imaging indexes of patients and their clinical conditions. Most of the former studies have focused on the relationship between pulmonary CT scan findings and the clinical condition of patients, and some reported paradoxical results but very few studies tried to evaluate quantitative imaging indexes and clinical conditions of patients. To date, very few studies have evaluated the use of CT severity score in patients with COVID-19 infection and no studies have been conducted in Iran. In the present study, regarding the importance and prevalence of COVID-19 infection in Iran and also regarding the high values of investigating the clinical conditions and their relationships with imaging characteristics, we aimed to evaluate and investigate the relationship between $\mathrm{CT}$ severity score and blood oxygen level by the time of admission in patients with COVID-19 infection.

\section{Methods And Material}

This is a cross-sectional study performed in 2020 in Isfahan, Iran on patients with COVID-19 infection. The research committee of Isfahan University of Medical Sciences has approved this study and the ethical committee has also confirmed it (Ethics code: IR.MUI. MED.REC.1399.301)

The inclusion criteria were patients over 14 years of age, confirming COVID-19 infection with PCR testing, patients who underwent pulmonary CT-scan, and measurements of capillary oxygen saturation using pulse oximetry at the time of admission, and having written informed consent to participate in the study. The exclusion criteria were incomplete data in the patient's electronic documents, atypical CT scans of the lung for COVID-19 infection, congenital heart and lung anomalies, any history of chronic heart and lung disease, and having severe hemoglobinopathy or anemia.

The study was performed by the census method. All of the patients referred to our medical center with a diagnosis of COVID-19 were included in the study based on inclusion and exclusion criteria. Demographic data of patients including age, sex, and presence of any past medical histories were collected. We collected all of the comorbidities and compared the CT-scan results and other clinical data among patients with or without these comorbidities.

The pulmonary CT scan was performed for all patients as following: all subjects underwent CT scan of the lung with slice thickness $=0.625-5 \mathrm{~mm}$ during hospitalization by Hitachi 32-MDCT device and were in supine and deep inspiration. The CT scan images were then reconstructed. Two experienced radiologists with a specialized board in the field of lung imaging simultaneously examined the pulmonary CT scan of all patients and calculated the CT scan score for patients (an experienced radiologist is a radiologist who has at least three years of experience in the field of body imaging). In cases of disagreement, they asked for help from a third radiologist who has more experience in body imaging (at least 10 years).

To calculate the $\mathrm{CT}$ severity score according to the anatomical structure of the lung, 18 segments of both lungs were divided into 20 separate areas so that the apicoposterior segment of the left upper lung was divided into the apical and posterior regions and the antromediobasal segment of the left lung was also divided into the anterior and basal regions. The radiologists examined the presence and spread of opacity in all 20 areas and scored each area as 0,1 , and 2 for $0 \%$, below $50 \%$, and above $50 \%$, respectively. The final score was obtained from the sum of points in each of the 20 areas and was considered as a CT severity score, which had a range of $0-40$.

The capillary blood oxygen saturation was also collected from documents of all patients. Hypoxia was measured by capillary blood saturation using pulse oximetry and hypoxia was defined as oxygen levels $<95 \%$. It should also be noted that the time interval between performing pulse oximetry and pulmonary $\mathrm{CT}$ scan in patients was a maximum of one day. Data were collected and analyzed to investigate the relationship between CT severity score and patient's capillary blood oxygen saturations using SPSS software version 23. Independent $t$-test, Mann-Whitney $U$ test, and Pearson correlation coefficient were performed at an error level of $5 \%$.

\section{Results}

A total number of 270 patients diagnosed with COVID-19 infection entered the study. The mean age of patients was $60.72 \pm 16.74$ years (25-97 years). The initial measurements of blood oxygen levels showed that mean blood oxygen saturation was $89.65 \pm 8.09 \%$ (40-100\%). Analysis of the CT severity score showed that the mean score in patients was $15.16 \pm 8.08(10-40)$.

We also indicated that a total number of 27 patients had hypoxia by the time of admission and these patients had significantly higher CT severity scores based on independent $t$-test ( $p=0.001)$. The mean CT severity score in patients with hypoxia was $18.14 \pm 7.43$ and the mean CT severity score in patients without hypoxia was $12.64 \pm 7.25$.

We evaluated CT severity score and blood oxygen saturation in patients based on gender and indicated that the mean CT severity score was significantly lower in male patients (Table 1).

Table 1: Analysis of CT severity score and oxygen saturation in patients based on gender

\begin{tabular}{lllll}
\hline Variable & & Mean & $\begin{array}{c}\text { Standard } \\
\text { deviation }\end{array}$ & p-value \\
\hline Age & Female & 61.16 & 16.36 & 0.73 \\
& Male & 60.47 & 17.07 & \\
Oxygen saturation & Female & 88.47 & 10.46 & 0.056 \\
& Male & 90.55 & 5.50 & \\
CT severity score & Female & 16.82 & 8.35 & 0.003 \\
& Male & 13.91 & 7.6 & \\
\hline
\end{tabular}


Table 2: Frequency distribution of various underlying diseases and their relationship with oxygen saturation

\begin{tabular}{|c|c|c|c|c|}
\hline \multirow{2}{*}{$\begin{array}{l}\text { Underlying disease } \\
\text { Diabetes mellitus }\end{array}$} & \multirow{2}{*}{$\begin{array}{l}\text { Frequency } \\
(N(\%))\end{array}$} & \multicolumn{2}{|c|}{$\begin{array}{l}\text { Oxygen saturation } \\
\text { (mean } \pm S D)\end{array}$} & \multirow{2}{*}{$\frac{p \text {-value }}{0.001^{*}}$} \\
\hline & & Without & $90.93 \pm 5.75$ & \\
\hline & & With & $86.55 \pm 11.45$ & \\
\hline \multirow[t]{2}{*}{ Hypertension } & $87(32.2)$ & Without & $91.19 \pm 5.54$ & $0.001 *$ \\
\hline & & With & $86.36 \pm 11.17$ & \\
\hline \multirow{2}{*}{$\begin{array}{l}\text { Chronic heart } \\
\text { disease }\end{array}$} & $46(17.0)$ & Without & $89.87 \pm 8.34$ & 0.06 \\
\hline & & With & $88.53 \pm 6.71$ & \\
\hline \multirow[t]{2}{*}{ Chronic renal failure } & $7(2.6)$ & Without & $89.71 \pm 8.03$ & 0.90 \\
\hline & & With & $87.14 \pm 10.65$ & \\
\hline \multirow{2}{*}{$\begin{array}{l}\text { Chronic lymphocytic } \\
\text { leukemia }\end{array}$} & $1(0.4)$ & Without & $89.66 \pm 8.10$ & 0.40 \\
\hline & & With & $87.00 \pm 0.0$ & \\
\hline \multirow[t]{2}{*}{ Cholesterolemia } & $15(5.6)$ & Without & $89.67 \pm 8.18$ & 0.53 \\
\hline & & With & $89.20 \pm 6.66$ & \\
\hline \multirow{2}{*}{$\begin{array}{l}\text { Interstitial lung } \\
\text { disease }\end{array}$} & $2(0.7)$ & Without & $89.64 \pm 8.12$ & 0.78 \\
\hline & & With & $90.50 \pm 3.53$ & \\
\hline \multirow[t]{2}{*}{ COPD } & $9(3.3)$ & Without & $89.83 \pm 8.02$ & $0.03^{*}$ \\
\hline & & With & $84.22 \pm 8.87$ & \\
\hline \multirow[t]{2}{*}{ Gastric cancer } & $1(0.4)$ & Without & $89.65 \pm 8.11$ & 0.56 \\
\hline & & With & $89.00 \pm 0.0$ & \\
\hline \multirow[t]{2}{*}{ Thalassemia } & $2(0.7)$ & Without & $89.62 \pm 8.12$ & 0.59 \\
\hline & & With & $92.50 \pm 0.70$ & \\
\hline \multirow[t]{2}{*}{ Asthma } & $5(1.8)$ & Without & $89.67 \pm 8.09$ & 0.53 \\
\hline & & With & $82.00 \pm 0.0$ & \\
\hline \multirow[t]{2}{*}{ Pulmonary infection } & $1(0.4)$ & Without & $89.66 \pm 8.11$ & 0.72 \\
\hline & & With & $89.00 \pm 7.96$ & \\
\hline \multirow[t]{2}{*}{ Down syndrome } & $1(0.4)$ & Without & $89.64 \pm 8.11$ & 0.72 \\
\hline & & With & $90.00 \pm 0.0$ & \\
\hline \multirow[t]{2}{*}{ Alzheimer's } & $1(0.4)$ & Without & $89.64 \pm 8.11$ & 0.72 \\
\hline & & With & $90.00 \pm 0.0$ & \\
\hline \multirow[t]{2}{*}{ Parkinson's } & $1(0.4)$ & Without & $89.64 \pm 8.11$ & 0.72 \\
\hline & & With & $90.00 \pm 0.0$ & \\
\hline \multirow[t]{2}{*}{ Hyperthyroidism } & $1(0.4)$ & Without & $89.64 \pm 8.11$ & 0.87 \\
\hline & & With & $92.00 \pm 0.0$ & \\
\hline \multirow[t]{2}{*}{ Total } & $137(50.7)$ & Without & $91.82 \pm 5.57$ & $<0.001^{*}$ \\
\hline & & With & $78.52 \pm 9.52$ & \\
\hline
\end{tabular}

Analysis of demographic data indicated that $50.7 \%$ of patients had at least one previous medical disease. Hypertension (32.2\%) and diabetes mellitus (29.6\%) were most common among patients. Investigating the relationship between underlying diseases and oxygen saturation showed that diabetes mellitus ( $p=0.001)$, hypertension $(p=0.001)$, and chronic obstructive pulmonary disease (COPD) $(p=0.03)$ and totally, having an underlying disease $(p<0.001)$ were significantly associated with decreased oxygen saturation in patients (Table 2).

We also showed that patients with hypertension $(p=0.001)$ and patients with the previous medical disease $(p=0.01)$ had significantly higher CT severity scores than others. These data are also summarized in Table 3.

Based on Pearson's correlation test, we showed that there was a significant reverse relationship between $\mathrm{CT}$ severity score and
Table 3: Relationship between underlying diseases and CT severity score

\begin{tabular}{|c|c|c|c|c|}
\hline Underlying disease & $\begin{array}{l}\text { Frequency } \\
(N(\%))\end{array}$ & $\begin{array}{l}\text { CT severit } \\
\text { (mean } \pm\end{array}$ & $\begin{array}{l}\text { y score } \\
\text { SD) }\end{array}$ & $p$-value \\
\hline \multirow[t]{2}{*}{ Diabetes mellitus } & $80(29.6)$ & Without & $14.30 \pm 7.89$ & 0.09 \\
\hline & & With & $17.21 \pm 8.20$ & \\
\hline \multirow[t]{2}{*}{ Hypertension } & $87(32.2)$ & Without & $13.97 \pm 7.53$ & $0.005^{*}$ \\
\hline & & With & $17.65 \pm 8.64$ & \\
\hline \multirow[t]{2}{*}{ Chronic heart disease } & $46(17.0)$ & Without & $15.15 \pm 8.23$ & 0.85 \\
\hline & & With & $15.19 \pm 7.40$ & \\
\hline \multirow[t]{2}{*}{ Chronic renal failure } & $7(2.6)$ & Without & $15.13 \pm 8.13$ & 0.72 \\
\hline & & With & $16.14 \pm 6.03$ & \\
\hline \multirow{2}{*}{$\begin{array}{l}\text { Chronic lymphocytic } \\
\text { leukemia }\end{array}$} & $1(0.4)$ & Without & $15.20 \pm 8.07$ & 0.22 \\
\hline & & With & $5.00 \pm 0.0$ & \\
\hline \multirow[t]{2}{*}{ Cholesterolemia } & $15(5.6)$ & Without & $15.06 \pm 8.14$ & 0.36 \\
\hline & & With & $16.80 \pm 6.90$ & \\
\hline \multirow{2}{*}{$\begin{array}{l}\text { Interstitial lung } \\
\text { disease }\end{array}$} & $2(0.7)$ & Without & $15.10 \pm 8.02$ & 0.40 \\
\hline & & With & $23.00 \pm 15.15$ & \\
\hline \multirow[t]{2}{*}{ COPD } & $9(3.3)$ & Without & $15.19 \pm 8.11$ & 0.98 \\
\hline & & With & $14.55 \pm 7.56$ & \\
\hline \multirow[t]{2}{*}{ Gastric cancer } & $1(0.4)$ & Without & $15.15 \pm 8.09$ & 0.82 \\
\hline & & With & $17.00 \pm 0.0$ & \\
\hline \multirow[t]{2}{*}{ Thalassemia } & $2(0.7)$ & Without & $15.17 \pm 8.10$ & 0.66 \\
\hline & & With & $13.00 \pm 5.65$ & \\
\hline \multirow[t]{2}{*}{ Asthma } & $5(1.8)$ & Without & $15.13 \pm 8.14$ & 0.46 \\
\hline & & With & $16.80 \pm 3.49$ & \\
\hline \multirow[t]{2}{*}{ Pulmonary infection } & $1(0.4)$ & Without & $15.20 \pm 8.07$ & 0.82 \\
\hline & & With & $5.00 \pm 0.0$ & \\
\hline \multirow[t]{2}{*}{ Down syndrome } & $1(0.4)$ & Without & $15.20 \pm 8.07$ & 0.19 \\
\hline & & With & $3.00 \pm 0.0$ & \\
\hline \multirow[t]{2}{*}{ Alzheimer's } & $1(0.4)$ & Without & $15.20 \pm 8.06$ & 0.19 \\
\hline & & With & $3.00 \pm 0.0$ & \\
\hline \multirow[t]{2}{*}{ Parkinson's } & $1(0.4)$ & Without & $15.18 \pm 8.08$ & 0.46 \\
\hline & & With & $9.00 \pm 0.0$ & \\
\hline \multirow[t]{2}{*}{ Hyperthyroidism } & $1(0.4)$ & Without & $15.14 \pm 8.09$ & 0.44 \\
\hline & & With & $20.00 \pm 0.0$ & \\
\hline \multirow[t]{2}{*}{ Total } & $137(50.7)$ & Without & $13.65 \pm 7.61$ & $0.01^{*}$ \\
\hline & & With & $16.62 \pm 8.28$ & \\
\hline
\end{tabular}

*Significant based on the Mann-Whitney $U$ test; COPD, chronic obstructive pulmonary disease

oxygen saturation ( $r=-0.44, p<0.001)$. This correlation was also significant among patients with underlying diseases $(r=-0.50$, $p<0.001)$ and among patients with no underlying diseases $(r=-0.28, p=0.01)$. On the other hand, no significant correlations were detected between oxygen saturation and age $(p=0.19)$.

\section{Discussion}

In the present study, we showed that patients with hypoxia had significantly higher CT severity score. We also showed that diabetes mellitus, hypertension, COPD and totally, having an underlying disease were significantly associated with decreased oxygen saturation. Furthermore, patients with hypertension and previous medical disease have significantly higher CT severity score. Our data also showed that there was a significant reverse relationship between CT severity score and oxygen saturation, which has pivotal 
clinical importance. These data emphasize the relationship between CT severity score and clinical condition of patients with COVID-19 infections. So far, some previous studies have discussed this issue but paradoxical results were also reported.

In a study by Yang et al. in China, quantitative and semiquantitative indicators of chest $\mathrm{CT}$ scan and their relationship to the clinical conditions of patients were evaluated. They investigated CT scan findings of 102 patients with COVID-19 infection and showed that the total CT severity score was significantly higher in patients with severe COVID-19 infections compared to mild cases. They also suggested that a CT severity score could be used to evaluate the severity of pulmonary involvement. ${ }^{17}$ It should also be mentioned that respiratory distress $\geq 30$ beats/min; resting blood oxygen saturation $\leq 93 \%$; or partial pressure of arterial blood oxygen $\left(\mathrm{PaO}_{2}\right)$ /oxygen concentration $\left(\mathrm{FiO}_{2}\right) \leq 300 \mathrm{~mm} \mathrm{Hg}$ were regarded as clinical severity criteria. These data are in line with our findings, showing higher CT severity scores in patients with hypoxia. Another study was performed by Zhao et al. on 101 cases of COVID-19 infection to investigate possible relationships between chest CT findings and clinical conditions of patients. They indicated that ground-glass opacities or mixed ground-glass opacities and consolidation and vascular enlargement were most common in patients but they also emphasized the use of quantitative and semiquantitative indicators for further evaluation or even prediction of the clinical condition of patients. They suggested that a CT severity score can help in the evaluation of the severity and extent of the disease. ${ }^{20}$ These data are in line with our findings. However, previous studies were not able to evaluate possible relationships between CT severity score and clinical characteristics of patients. We also showed that patients with hypertension and patients with previous medical disease had significantly higher $\mathrm{CT}$ severity scores which is another key point of the current study.

The beneficial use of CT severity score in patients with COVID19 infections has been also indicated in some other studies ${ }^{21}$ but they could not determine any specific clinical feature in patients that were correlated with this score. Another study was conducted in China by Xiong et al. on 42 patients with COVID-19 infection to determine any possible relationship between chest CT scan findings and clinical parameters. They indicated that COVID-19 infection usually presents with ground glass opacities in pulmonary CT scan, which is also correlated with higher C-reactive protein, erythrocyte sedimentation rate, and lactate dehydrogenase. However, they could not suggest any related clinical feature with the severity of CT scan findings. ${ }^{22}$ We believe that such variations could be due to differences in the studied populations. So far, the possible relation between CT severity score and oxygen saturation in patients with COVID-19 has not been investigated in the studies. Here for the first time, we showed a significant relationship between oxygen saturation and CT severity score. This might be considered a key finding for patients with COVID-19 infection to evaluate or even predict their clinical conditions.

Fang et al. compared the results of CT severity score to results of reverse transcription-polymerase chain reaction (RT-PCR) in 51 patients with COVID-19 infection. They showed that the sensitivity of chest CT was greater than that of RT-PCR ( $98 \%$ vs $71 \%$, respectively; $p<0.001)$ and patients with higher CT severity scores had more severe infection in the following days. ${ }^{23}$ These results indicate that a higher CT severity score is related to more severe clinical conditions in patients. We also believe that associations between higher CT severity scores and hypoxia could justify this issue. In a recent study by Yang et al., they tried to describe the clinical characteristics and imaging manifestations of 149 hospitalized patients with confirmed COVID-19 infection. They showed that multifocal peripheral ground glass or mixed opacity with predominance in the lower lung was the most common finding in patients and the severity of such findings was associated with the severity of the infection in some cases. ${ }^{24}$ Cheng et al. also evaluated CT scan findings of patients suspicious of COVID-19 infections and reported that imaging patterns of multifocal, peripheral, pure ground-glass opacity (GGO), mixed $\mathrm{GGO}$, or consolidation with a slight predominance in the lower lung in the first week of illness could be considered as findings which are highly suspicious of COVID-19. But they were not able to clarify if there were any correlations between such CT scan findings and clinical features of patients. ${ }^{25}$ As we showed, previous studies could not discuss if there were any relationships between clinical conditions in COVID-19 infected patients and CT severity score. The limitations of this study were that this study could have unknown potential confounders, we used the data that were originally collected for these purposes, not all the relevant information, and we had also an inferior level of evidence compared with prospective studies. We also had a restricted study population compared to some former studies and therefore, suggest that more studies on larger populations should be performed. We also suggest that physicians should consider CT severity score as a useful method to evaluate patient's conditions.

\section{Conclusion}

Here we showed that patients with hypoxia have significantly higher CT severity scores. We showed that there was a significant reverse relationship between $\mathrm{CT}$ severity score and oxygen saturation which has great clinical importance. Furthermore, our data showed that previous medical history could also play an important role in CT severity score. These data were in line with some of the previous studies but some other studies could not discuss them. We suggest that physicians should pay more attention to $\mathrm{CT}$ severity scores in patients with COVID-19 infection.

\section{ACKNOWLEDgMentS}

Marzieh Aalinezhad: Substantial contributions to the conception or design of the work, drafting the work, final approval of the version to be published, and agreement to be accountable for all aspects of the work in ensuring that questions related to the accuracy or integrity of any part of the work are appropriately investigated and resolved.

Fariba Alikhani: Substantial contributions to the conception or design of the work, revising the work critically for important intellectual content, final approval of the version to be published, and agreement to be accountable for all aspects of the work in ensuring that questions related to the accuracy or integrity of any part of the work are appropriately investigated and resolved.

Pooya Akbari: Design of the work, revising the work critically for important intellectual content, final approval of the version to be published, and agreement to be accountable for all aspects of the work in ensuring that questions related to the accuracy or integrity of any part of the work are appropriately investigated and resolved.

Mostafa H Rezaei: Analysis or interpretation of data for the work, drafting the work, final approval of the version to be published, and agreement to be accountable for all aspects of the work in ensuring that questions related to the accuracy or integrity of any part of the work are appropriately investigated and resolved. 
Samira Soleimani: Design of the work, revising the work critically for important intellectual content, final approval of the version to be published, and agreement to be accountable for all aspects of the work in ensuring that questions related to the accuracy or integrity of any part of the work are appropriately investigated and resolved.

Atoosa Hakamifard: Substantial contributions to the conception or design of the work, drafting the work, final approval of the version to be published, and agreement to be accountable for all aspects of the work in ensuring that questions related to the accuracy or integrity of any part of the work are appropriately investigated and resolved.

\section{ORCID}

Marzieh Aalinezhad 이 https://orcid.org/0000-0002-9183-788X

Fariba Alikhani @ https://orcid.org/0000-0003-4904-1420

Pooya Akbari (1) https://orcid.org/0000-0003-2801-321X

Mostafa Haji Rezaei @ https://orcid.org/0000-0001-9798-1471

Samira Soleimani ↔ https://orcid.org/0000-0002-1568-6944

Atousa Hakamifard @ 1 https://orcid.org/0000-0001-9456-2239

\section{References}

1. Novel CPERE. The epidemiological characteristics of an outbreak of 2019 novel coronavirus diseases (COVID-19) in China. Zhonghua Liu Xing Bing Xue Za Zhi 2020;41(2):145-151. DOI: 10.3760/ cma.j.issn.0254-6450.2020.02.003.

2. Van Der Hoek L, Pyrc K, Jebbink MF, Vermeulen-Oost W, Berkhout RJ, Wolthers KC, et al. Identification of a new human coronavirus. Nat Med 2004;10(4):368-373. DOI: 10.1038/nm1024.

3. Cao J, Tu W-J, Cheng W, Yu L, Liu Y-K, Hu X, et al. Clinical features and short-term outcomes of 102 patients with corona virus disease 2019 in Wuhan, China. Clin Infect Dis 2020;71(15):748-755. DOI: 10.1093/ $\mathrm{cid} / \mathrm{ciaa243.}$

4. Andersen KG, Rambaut A, Lipkin WI, Holmes EC, Garry RF. The proximal origin of SARS-CoV-2. Nat Med 2020;26(4):450-452. DOI: 10.1038/s41591-020-0820-9.

5. Wang W, Xu Y, Gao R, Lu R, Han K, Wu G, et al. Detection of SARS-CoV-2 in different types of clinical specimens. JAMA 2020;323(18): 1843-1844. DOI: 10.1001/jama.2020.3786.

6. Spinelli A, Pellino G. COVID-19 pandemic: perspectives on an unfolding crisis. Br J Surg 2020;107(7):785-787. DOI: 10.1002/bjs.11627.

7. Lythgoe MP, Middleton P. Ongoing clinical trials for the management of the COVID-19 pandemic. Trends Pharmacol Sci 2020;41(6):363-382. DOI: 10.1016/j.tips.2020.03.006.

8. Han R, Huang L, Jiang H, Dong J, Peng H, Zhang D. Early clinical and CT manifestations of coronavirus disease 2019 (COVID-19) pneumonia. AJR Am J Roentgenol 2020;215(2):1-6. DOI: 10.2214/AJR.20.22961.

9. Cascella M, Rajnik M, Cuomo A, Dulebohn SC, Di Napoli R. Features, evaluation and treatment coronavirus (COVID-19). StatPearls [Internet]. Treasure Island, FL: StatPearls Publishing; 2020.

10. Rodriguez-Morales AJ, Cardona-Ospina JA, Gutiérrez-Ocampo E, Villamizar-Peña R, Holguin-Rivera Y, Escalera-Antezana JP, et al. Clinical, laboratory and imaging features of COVID-19: a systematic review and meta-analysis. Travel Med Infect Dis 2020;34:101623. DOI: 10.1016/j.tmaid.2020.101623.

11. Li W, Cui H, Li K, Fang Y, Li S. Chest computed tomography in children with COVID-19 respiratory infection. Pediatr Radiol 2020;50(6): 796-799. DOI: 10.1007/s00247-020-04656-7.

12. Salehi S, Abedi A, Balakrishnan S, Gholamrezanezhad A. Coronavirus disease 2019 (COVID-19): a systematic review of imaging findings in 919 patients. AJR Am J Roentgenol 2020;215(1):87-93. DOI: 10.2214/ AJR.20.23034.

13. Wang W, Gao R, Zheng Y, Jiang L. COVID-19 with spontaneous pneumothorax, pneumomediastinum and subcutaneous emphysema. J Travel Med 2020;27(5):taaa062. DOI: 10.1093/jtm/taaa062.

14. Ye Z, Zhang Y, Wang Y, Huang Z, Song B. Chest CT manifestations of new coronavirus disease 2019 (COVID-19): a pictorial review. Eur Radiol 2020;30(8):4381-4389. DOI: 10.1007/s00330-020-06801-0.

15. Ali TF, Tawab MA, ElHariri MA. CT chest of COVID-19 patients: what should a radiologist know? Egypt J Radiol Nucl Med 2020;51(1):1-6. DOI: 10.1186/s43055-020-00245-8.

16. Bankier AA, Janata K, Fleischmann D, Kreuzer S, Mallek R, Frossard $M$, et al. Severity assessment of acute pulmonary embolism with spiral CT: evaluation of two modified angiographic scores and comparison with clinical data. J Thorac Imaging 1997;12(2):150-158. DOI: 10.1097/00005382-199704000-00012.

17. Yang R, Li X, Liu H, Zhen Y, Zhang X, Xiong Q, et al. Chest CT severity score: an imaging tool for assessing severe COVID-19. Radiol Cardiothorac Imaging 2020;2(2):e200047. DOI: 10.1148/ ryct.2020200047.

18. Liu Y, Yan L-M, Wan L, Xiang T-X, Le A, Liu J-M, et al. Viral dynamics in mild and severe cases of COVID-19. Lancet Infect Dis 2020;20(6): 656-657. DOI: 10.1016/S1473-3099(20)30232-2.

19. Xie J, Covassin N, Fan Z, Singh P, Gao W, Li G, et al. Association between hypoxemia and mortality in patients with COVID-19. Mayo Clin Proc 2020;95(6):1138-1147. DOI: 10.1016/j.mayocp.2020.04.006.

20. Zhao W, Zhong Z, Xie X, Yu Q, Liu J. Relation between chest CT findings and clinical conditions of coronavirus disease (COVID-19) pneumonia: a multicenter study. AJR Am J Roentgenol 2020;214(5):1072-1077. DOI: 10.2214/AJR.20.22976.

21. Li K, Wu J, Wu F, Guo D, Chen L, Fang Z, et al. The clinical and chest CT features associated with severe and critical COVID-19 pneumonia. Invest Radiol 2020;55(6):327-331. DOI: 10.1097/ rli.0000000000000672.

22. Xiong Y, Sun D, Liu Y, Fan Y, Zhao L, Li X, et al. Clinical and highresolution CT features of the COVID-19 infection: comparison of the initial and follow-up changes. Invest Radiol 2020;55(6):332-339. DOI: 10.1097/RLI.0000000000000674.

23. Fang $Y$, Zhang $H$, Xie J, Lin M, Ying L, Pang $P$, et al. Sensitivity of chest CT for COVID-19: comparison to RT-PCR. Radiology 2020;296(2): E115-E117. DOI: 10.1148/radiol.2020200432.

24. Yang W, Cao Q, Qin L, Wang X, Cheng Z, Pan A, et al. Clinical characteristics and imaging manifestations of the 2019 novel coronavirus disease (COVID-19): a multi-center study in Wenzhou city, Zhejiang, China. J Infect 2020;80(4):388-393. DOI: 10.1016/j. jinf.2020.02.016.

25. Cheng Z, Lu Y, Cao Q, Qin L, Pan Z, Yan F, et al. Clinical features and chest CT manifestations of coronavirus disease 2019 (COVID-19) in a single-center study in Shanghai, China. AJR Am J Roentgenol 2020;215(1):1-6. DOI: 10.2214/AJR.20.22959. 